\title{
Vortex states in hole-doped iron-pnictide superconductors
}

\author{
Yi Gao ${ }^{1}$, Huai-Xiang Huang ${ }^{1,2}$, Chun $\mathrm{Chen}^{3}$, C. S. Ting ${ }^{1,3}$, and Wu-Pei Su${ }^{1}$ \\ ${ }^{1}$ Department of Physics and Texas Center for Superconductivity, \\ University of Houston, Houston, Texas, 77204, USA \\ ${ }^{2}$ Department of Physics, Shanghai University, Shanghai, 200444, China \\ ${ }^{3}$ Department of Physics, Fudan University, Shanghai, 200433, China
}

\begin{abstract}
Based on a phenomenological model with competing spin-density-wave (SDW) and extended $s$-wave superconductivity, the vortex states in $\mathrm{Ba}_{1-x} \mathrm{~K}_{x} \mathrm{Fe}_{2} \mathrm{As}_{2}$ are investigated by solving Bogoliubov-de Gennes equations. Our result for the optimally doped compound without induced SDW is in qualitative agreement with recent scanning tunneling microscopy experiment. We also propose that the main effect of the SDW on the vortex states is to reduce the intensity of the in-gap peak in the local density of states and transfer the spectral weight to form additional peaks outside the gap.
\end{abstract}

PACS numbers: 74.70.Xa, 74.25.Ha, 74.25.Op

The recent discovery of the iron-pnictide superconductors [1], whose parent compounds exhibit long-range antiferromagnetic (AF) or spin-density-wave (SDW) order similar to the cuprates [2], provides another promising group of materials for studying the interplay between magnetism and superconductivity (SC). Especially, the hole-doped pnictide superconductors, like $\mathrm{Ba}_{1-x} \mathrm{~K}_{x} \mathrm{Fe}_{2} \mathrm{As}_{2}$ [3], have emerged as one of the most important systems due to the availability of large homogeneous single crystals. The phase diagram [4] for these materials indicates that the parent compound, upon cooling through $\mathrm{T}_{N} \sim 140 \mathrm{~K}[3,5]$, develops a static SDW order. By increasing the doping of potassium, the SDW order is suppressed and the SC order emerges as the temperature (T) falls below $\mathrm{T}_{c}$. The SDW and SC orders coexist in the underdoped samples [4]. By further increasing the potassium concentration to the optimally doped regime, the SDW order disappears. These experimental results provide compelling evidence for strong competition between the SDW and SC orders.

Another key issue here is the superconducting pairing symmetry. Experimental results on the pairing symmetry remain highly controversial, leaving the perspectives ranging from nodeless [6, 7] to nodal gap structure [8 10 ]. Although evidence for a nodal gap has been accumulated in $\mathrm{LaFePO}$ [9] and $\mathrm{Ba}\left(\mathrm{FeAs}_{1-x} \mathrm{P}_{x}\right)_{2}$ [10] systems, in the $\mathrm{K}$ - and Co-doped 122-family of iron pnictides, the experimental data points to the existence of isotropic gaps, especially in the optimally doped samples [7]. Theoretically it was suggested that the pairing may be established via inter-pocket scattering of electrons between the hole pockets (around the $\Gamma$ point) and electron pockets (around the $M$ point), leading to the so-called extended $s$-wave (ES) pairing symmetry $\left(\Delta_{\mathbf{k}} \sim \cos k_{x}+\cos k_{y}\right)$ [11].

In this regard, investigating the vortex states in the iron-pnictides can provide useful information on the interplay between the SDW and SC, as well as the pairing symmetry. Recent scanning tunneling microscopy (STM) measurement on $\mathrm{Ba}_{1-x} \mathrm{~K}_{x} \mathrm{Fe}_{2} \mathrm{As}_{2}$ [12] has revealed, for the first time in the iron-pnictides, the existence of the Andreev bound states inside the vortex core with a systematic evolution: a single conductance peak appears at a negatively-biased voltage at the vortex center, which gradually evolves into two sub-peaks when moving away from the center, with a dominant spectral weight at negative bias. This negatively-biased conductance peak has not been observed in electron-doped $\mathrm{Ba}\left(\mathrm{Fe}_{1-x} \mathrm{Co}_{x}\right)_{2} \mathrm{As}_{2}$ [13] and is beyond current theoretical predictions where a peak appears at positive bias in a two-orbital model 14 and at zero bias in a five-orbital model [12]. Therefore, it is important to develop a sound theory for the vortex states in the iron-pnictide superconductors.

In this work, we adopt a phenomenological model with competing SDW and extended $s$-wave superconductivity (ESSC) to study the vortex states in $\mathrm{Ba}_{1-x} \mathrm{~K}_{x} \mathrm{Fe}_{2} \mathrm{As}_{2}$ from the local density of states (LDOS). We show that the evolution of the resonance peak in the calculated LDOS is in qualitative agreement with STM experiment. Moreover, the effect of SDW on the vortex states is also discussed, which we predict to be measurable by future experiments.

We begin with a phenomenologically effective twoorbital model on a two-dimensional (2D) lattice which considers the asymmetry of the As atoms above and below the Fe layer [15], where the onsite interactions are solely responsible for the SDW while the next-nearestneighbor (NNN) intraorbital attraction causes the ESSC. The effective mean-field Hamiltonian can be written as 16]

$$
\begin{gathered}
H=-\sum_{i j, \alpha \beta, \sigma} t_{i j, \alpha \beta}^{\prime} c_{i \alpha \sigma}^{\dagger} c_{j \beta \sigma} \\
+\sum_{j \beta \sigma}\left[-\mu+U n_{j \beta \bar{\sigma}}+\left(U-2 J_{H}\right) n_{j \bar{\beta} \bar{\sigma}}\right. \\
\left.+\left(U-3 J_{H}\right) n_{j \bar{\beta} \sigma}\right] c_{j \beta \sigma}^{\dagger} c_{j \beta \sigma}
\end{gathered}
$$




$$
+\sum_{i j, \alpha \beta}\left(\Delta_{i j, \alpha \beta} c_{i \alpha \uparrow}^{\dagger} c_{j \beta \downarrow}^{\dagger}+\text { H.c. }\right) .
$$

Here $i, j$ are the site indices, $\alpha, \beta=1,2$ are the orbital indices, $\sigma$ represents the spin, $\mu$ is the chemical potential, and $n_{j \beta \sigma}=\left\langle c_{j \beta \sigma}^{\dagger} c_{j \beta \sigma}\right\rangle$ is the electron density. $U$ and $J_{H}$ are the onsite intraorbital Hubbard repulsion and Hund coupling, respectively. Here we have the interorbital Coulomb interaction $U^{\prime}=U-2 J_{H}$ according to symmetry [17]. $\Delta_{i j, \alpha \beta}=\delta_{\alpha \beta} \frac{V}{2}\left(\left\langle c_{j \beta \downarrow} c_{i \alpha \uparrow}\right\rangle-\left\langle c_{j \beta \uparrow} c_{i \alpha \downarrow}\right\rangle\right)$ is the intraorbital spin-singlet ES bond order parameter, where $V$ is the NNN intraorbital attraction. The reason we adopt this model is its ability [16] to qualitatively account for the doping evolution of the Fermi surface and the asymmetry in the SC coherent peaks as observed by the angle resolved photo-emission spectroscopy 18] and STM [19] experiments on $\mathrm{Ba}\left(\mathrm{Fe}_{1-x} \mathrm{Co}_{x}\right)_{2} \mathrm{As}_{2}$. In the presence of a magnetic field $B$ perpendicular to the plane, the hopping integral can be expressed as $t_{i j, \alpha \beta}^{\prime}=t_{i j, \alpha \beta} \exp \left[i \frac{\pi}{\Phi_{0}} \int_{j}^{i} \mathbf{A}(\mathbf{r}) \cdot d \mathbf{r}\right]$, where $\Phi_{0}=h c / 2 e$ is the superconducting flux quantum, and $\mathbf{A}=(-B y, 0,0)$ is the vector potential in the Landau gauge. Following Ref. [15], we have

$t_{i j, \alpha \beta}= \begin{cases}t_{1} & \alpha=\beta, i=j \pm \hat{x}(\hat{y}), \\ \frac{1+(-1)^{j}}{2} t_{2}+\frac{1-(-1)^{j}}{2} t_{3} & \alpha=\beta, i=j \pm(\hat{x}+\hat{y}), \\ \frac{1+(-1)^{j}}{2} t_{3}+\frac{1-(-1)^{j}}{2} t_{2} & \alpha=\beta, i=j \pm(\hat{x}-\hat{y}), \\ t_{4} & \alpha \neq \beta, i=j \pm(\hat{x} \pm \hat{y}), \\ 0 & \text { otherwise. }\end{cases}$

The mean-field Hamiltonian (1) can be diagonalized by solving self-consistently the Bogoliubov-de Gennes (BdG) equations:

$$
\begin{gathered}
H=C^{\dagger} M C, \\
C^{\dagger}=\left(\cdots, c_{j 1 \uparrow}^{\dagger}, c_{j 1 \downarrow}, c_{j 2 \uparrow}^{\dagger}, c_{j 2 \downarrow}, \cdots\right),
\end{gathered}
$$

subject to the self-consistency conditions for the electron density and the ES bond order parameter: $n_{j \beta \uparrow}=$ $\sum_{k=1}^{L}\left|Q_{m-1 k}\right|^{2} f\left(E_{k}\right), n_{j \beta \downarrow}=1-\sum_{k=1}^{L}\left|Q_{m k}\right|^{2} f\left(E_{k}\right)$ and $\Delta_{i j, \beta \beta}=\frac{V}{2} \sum_{k=1}^{L}\left(Q_{m k}^{*} Q_{n k}+Q_{n+1 k}^{*} Q_{m-1 k}\right) f\left(E_{k}\right)$. Here $L=4 N_{x} N_{y}$, with $N_{x} / N_{y}$ being the number of lattice sites along $\hat{x} / \hat{y}$ direction of the $2 \mathrm{D}$ lattice. $m=$ $4\left(j_{y}+N_{y} j_{x}\right)+2 \beta, n=4\left(i_{y}+N_{y} i_{x}\right)+2 \alpha-1$ and $Q$ is a unitary matrix that satisfies $\left(Q^{\dagger} M Q\right)_{k p}=\delta_{k p} E_{k}$. Here we used $i=\left(i_{x}, i_{y}\right)$ and $j=\left(j_{x}, j_{y}\right)$, with $i_{x}, j_{x}=$ $0,1, \ldots, N_{x}-1$ and $i_{y}, j_{y}=0,1, \ldots, N_{y}-1$. The chemical potential $\mu$ is determined by the doping concentration $x$ through $\frac{1}{N_{x} N_{y}} \sum_{j \beta \sigma} n_{j \beta \sigma}=2-\frac{x}{2}$. The ES order parameter at site $j$ is $\Delta_{j \beta}^{\prime}=\left(\Delta_{j+\hat{x}+\hat{y} j, \beta \beta}^{\prime}+\right.$ $\left.\Delta_{j-\hat{x}-\hat{y} j, \beta \beta}^{\prime}+\Delta_{j+\hat{x}-\hat{y} j, \beta \beta}^{\prime}+\Delta_{j-\hat{x}+\hat{y} j, \beta \beta}^{\prime}\right) / 4$ where $\Delta_{i j, \beta \beta}^{\prime}=$ $\Delta_{i j, \beta \beta} \exp \left[i \frac{\pi}{\Phi_{0}} \int_{j}^{(i+j) / 2} \mathbf{A}(\mathbf{r}) \cdot d \mathbf{r}\right]$. The LDOS is given by
$\rho_{i}(\omega)=\sum_{k=1}^{L} \sum_{\alpha}\left[\left|Q_{n k}\right|^{2} \delta\left(\omega-E_{k}\right)+\left|Q_{n+1 k}\right|^{2} \delta\left(\omega+E_{k}\right)\right]$, the supercell technique is used to calculate the LDOS.

In our calculation, the magnitudes of the parameters are chosen as $t_{1-4}=1,0.4,-2,0.04$ [16], $U=3.7$, $V=-2$ and $T=10^{-4}$. Magnetic unit cells are introduced where each unit cell accommodates two superconducting flux quantum and the linear dimension is $N_{x} \times N_{y}=48 \times 24$, which is larger than the coherence length $\xi$ of the iron-pnictides [13]. Throughout the paper, the length and energy are measured in units of the Fe-Fe distance $a$ and $t_{1}$, respectively. In the following, we focus on two doping concentrations $x=0.4$ and 0.3 , corresponding to the optimally doped and underdoped compounds, respectively.

At $x=0.4$, first let us choose $J_{H}=0.2 U$ such that, at $B=0$, SDW is completely suppressed and the ES order parameter $\Delta_{j \beta}^{\prime}$ is homogeneous in real space. Figures 1(a) and 1(b) show the spatial variations of the reduced ES order parameter $\Delta_{j \beta}^{R}=\left|\Delta_{j \beta}^{\prime} / \Delta_{j \beta}^{\prime}(B=0)\right|$ and the electron density $n_{j}=\sum_{\beta \sigma} n_{j \beta \sigma}$ plotted on a $24 \times 24$ lattice. The vortex center is located at site $(11,12)$ and no $\mathrm{SDW}$ is induced. The reduced ES order parameter $\Delta_{j \beta}^{R}$ vanishes at the vortex center and starts to increase at the scale of the coherence length $\xi$ to its bulk value, but the increase is slower along the $\pi / 4$ and $3 \pi / 4$ directions with respect to the underlying lattice. On the other hand, the electron density $n_{j}$ is strongly enhanced at the vortex center which is compensated by a depletion of electrons around two lattice spacings away from the center, after which $n_{j}$ decays also at the scale of $\xi$ to its bulk value, with no obviously slow variations along the $\pi / 4$ and $3 \pi / 4$ directions. The zero-energy(ZE) LDOS plotted in Fig. 1(c) also peaks at site $(11,12)$ and has the same fourfold rotational symmetry (RS) as $\Delta_{j \beta}^{R}$. In order to reveal the spatial variation of LDOS modulated by the vortex, in Fig. 1(d) we plot the LDOS at four typical positions along the black cut in Fig. 1(c). As we can see, at the vortex center, there is a remarkable negative-energy (NE) in-gap peak located at $-0.125 \Delta$, which is precisely the same as observed in Ref. [12]. When moving away from the center, the peak will split into two in-gap peaks with a dominant spectral weight at negative energy. Finally, the LDOS evolves continuously into its bulk feature. The ingap peak and evolution of the LDOS clearly indicate the existence of the Andreev bound states inside the vortex core, consistent with Ref. [12].

In order to study the effect of induced SDW on the vortex states, we perform the calculation for $J_{H}=0.23 U$. Like the $J_{H}=0.2 U$ case, at $B=0, \mathrm{SDW}$ is completely suppressed and the ES order parameter $\Delta_{j \beta}^{\prime}$ is homogeneous in real space, but the vortex states are fundamentally different from those for $J_{H}=0.2 U$ and they are presented in Fig. 2. As shown in Fig. 2(a), the vortex center is still located at site $(11,12)$ where the reduced ES order parameter $\Delta_{j \beta}^{R}$ vanishes, but the size of the vortex core is slightly enlarged compared to the 


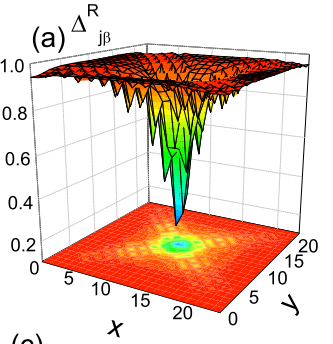

(c)

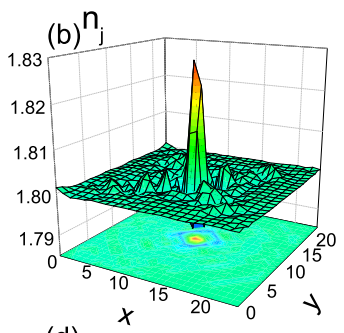

(d)
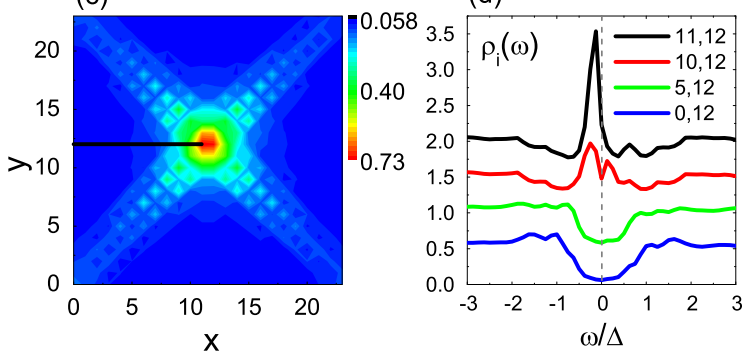

FIG. 1: (Color online) Spatial variations of (a) the reduced ES order parameter $\Delta_{j \beta}^{R}$, (b) electron density $n_{j}$, and (c) ZE LDOS map plotted on a $24 \times 24$ lattice. (d) The LDOS at four typical positions along the black cut in (c): at the vortex center $(11,12)$; within the vortex core while away from the center $(10,12)$; around the edge of a vortex $(5,12)$; and far outside a vortex $(0,12)$. The curves in (d) are displaced vertically for clarity and the gray dashed line indicates the position of zero energy.

$J_{H}=0.2 U$ case. The induced SDW order parameter defined as $M_{j}^{s}=(-1)^{j_{y}}\left(n_{j \uparrow}-n_{j \downarrow}\right)$ displayed in Fig. 2(b) reaches its maximum strength at the vortex center and decays at the scale of $\xi$ to zero into the superconducting region. More interestingly, the SDW order parameter has opposite polarity around two nearest-neighbor vortices along the $\hat{x}$ direction, thus doubling the period of the translational symmetry (TS) of the vortex lattice along this direction. Furthermore, with the induced SDW order, the electron density $n_{j}$ is only moderately enhanced near the vortex center with a depletion of electrons around the edge of the vortex core [see Fig. 2(c)]. The ZE LDOS in Fig. 2(d) also shows a slightly enlarged vortex core and the RS inside the core is reduced from fourfold to twofold due to the induced SDW order. Figures $2(\mathrm{e})$ and $2(\mathrm{f})$ are the spatial variations of the LDOS along the black and white cuts in Fig. 2(d), respectively. The spectra have only minor differences along the two cuts, mainly inside the vortex core and close to $\omega=0$ (indicated by the black arrow in Fig. 2(f)). At the vortex center, besides a NE in-gap peak at $-0.375 \Delta$, there is a small positive-energy in-gap peak at $0.125 \Delta$ which does not exist for $J_{H}=0.2 U$. In addition, the intensity of the NE peak is strongly reduced as compared to the $J_{H}=0.2 U$ case and the spectral weight is transferred to form additional peaks outside the gap as indicated by the
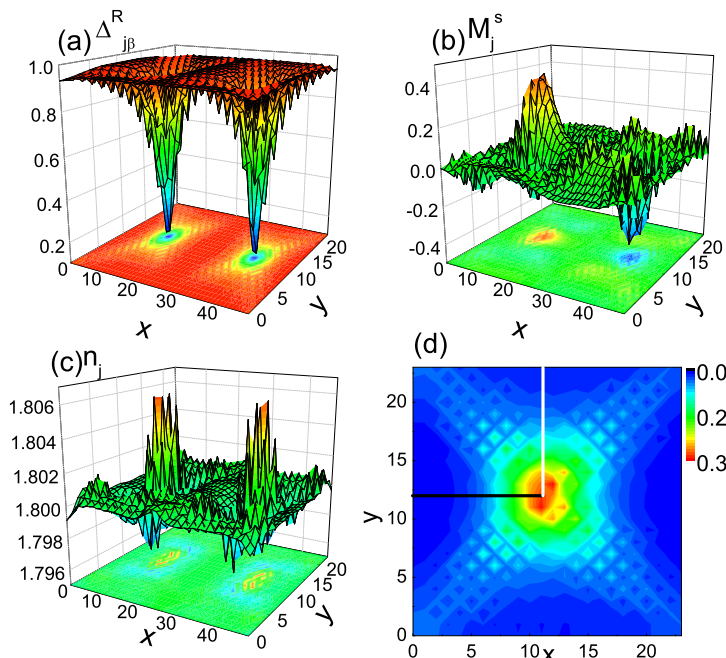

(d)
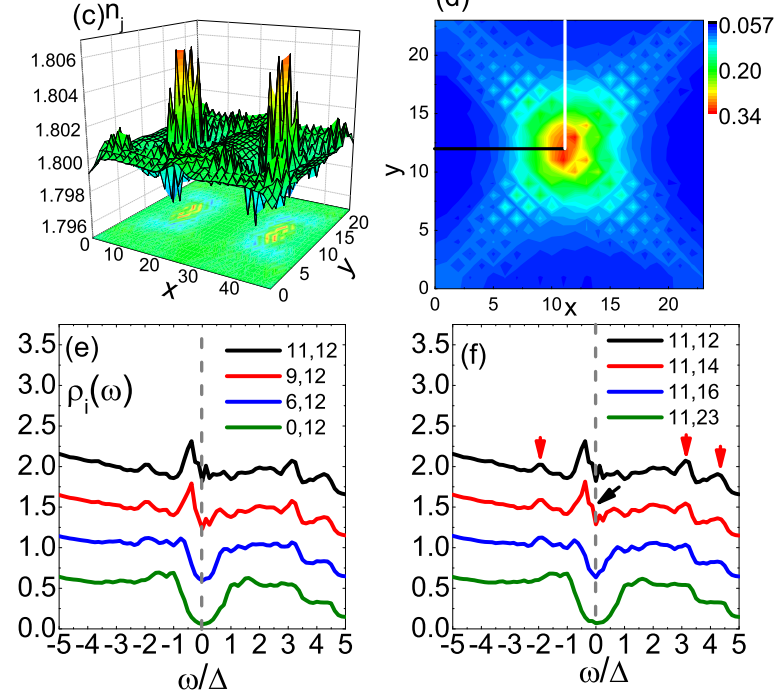

FIG. 2: (Color online) Spatial variations of (a) the reduced ES order parameter $\Delta_{j \beta}^{R}$, (b) staggered magnetization $M_{j}^{s}$, and (c) electron density $n_{j}$ plotted on a $48 \times 24$ lattice. (d) The ZE LDOS map plotted on a $24 \times 24$ lattice. (e) The LDOS at four typical positions along the black cut in (d). (f) is similar to (e), but is plotted along the white cut.

red arrows in Fig. 2(f). When moving away from the center, the intensities of all these peaks decrease and finally the LDOS evolves into its bulk feature. By comparing with the $J_{H}=0.2 U$ case, we can identify that those two in-gap peaks are due to the Andreev bound states while the others are due to the induced SDW order inside the core.

At $x=0.3$, we choose $J_{H}=0.32 U$ so that, at $B=0$, the $(\pi, 0)$ SDW coexists with the ESSC. The vortex states are plotted in Fig. 3. The reduced ES order parameter $\Delta_{j \beta}^{R}$ [see Fig. 3(a)] shows a $\hat{y}$-axis oriented stripe-like feature with a modulation period of $8 a$. The size of the vortex core is further enlarged and elongated along the $\hat{y}$ direction. Moreover, the two vortex cores are dragged towards each other along the $\hat{x}$ direction with the vortex centers located at sites $(15,12)$ and $(32,12)$, thus also doubling the period of the TS of the vortex lattice along this direction. The SDW order parameter $M_{j}^{s}$ shown in Fig. 3(b) behaves like nearly uniform stripes oscillating with a wavelength of $16 a$. The vortex core is pinned at one of the ridges of SDW stripes where the SDW order 

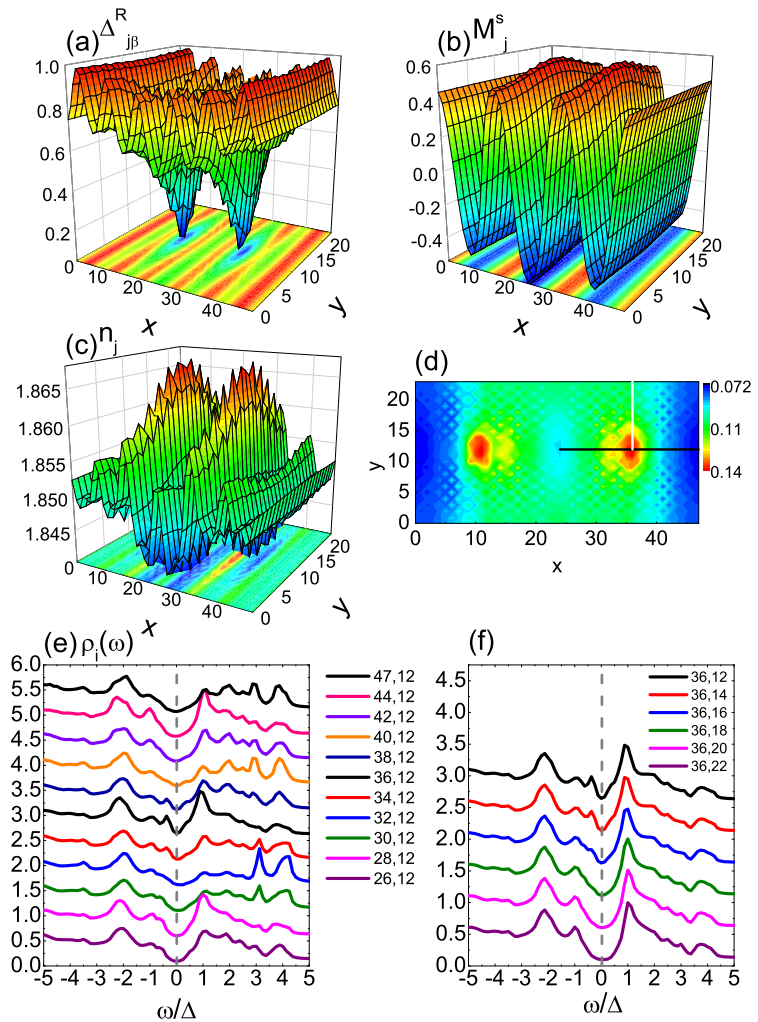

FIG. 3: (Color online) (a), (b), (c) and (d) are similar to Figs. 2(a), 2(b), 2(c) and 2(d), respectively. (e) The LDOS along the black cut in (d). (f) is similar to (e), but is plotted along the white cut.

is stronger than those at other sites. The spatial variation of the electron density $n_{j}$ also exhibits a quasi-onedimensional charge stripe behavior with a wavelength $8 a$, exactly half that of the SDW along the $\hat{x}$ direction [see Fig. 3 (c)]. The one-dimensional stripe-like modulations in $\Delta_{j \beta}^{R}, M_{j}^{s}$ and $n_{j}$ already exist at $B=0$, which are quite similar to the cuprates except for a doubling of the period from $4 a$ (for $\Delta_{j \beta}^{R}$ and $n_{j}$ ) and $8 a$ (for $M_{j}^{s}$ ) in the cuprates [20] to $8 a$ and $16 a$ in the iron-pnictides. The origin of such stripes could be understood in terms of the existence of a nesting wave vector $q_{A} \sim 0.125 \pi / a$ connecting the left (right) pieces of the inner and outer hole pockets around the $\Gamma$ point along the $k_{x}$ direction. For proper values of $U, J_{H}$ and doping, this wave vector would modulate $M_{j}^{s}$ with SDW stripes along the $\hat{x}$ direction with period $2 \pi / q_{A}=16 a$. The ZE LDOS in Fig. 3(d) also shows an enlarged vortex core, the doubling of the period of the TS of the vortex lattice along the $\hat{x}$ direction and the reduced RS from fourfold to twofold. Interestingly, although the two vortex cores are dragged towards each other, the ZE LDOS still peaks at sites $(11,12)$ and $(36,12)$, suggesting that even in the region where $\Delta_{j \beta}^{R} \neq 0$, there are ZE states contributing to the
LDOS. The spatial variations of the LDOS plotted in Figs. 3(e) and 3(f) show that at the ZE LDOS peak position, there is a NE in-gap peak at $-0.375 \Delta$, whose intensity is further reduced compared to that in Fig. $2(\mathrm{f})$ and the intensity decreases when moving away from the peak position, indicating that it is due to the Andreev bound states. There are also additional peaks outside the gap whose positions are similar to those marked by the red arrows in Fig. 2(f). Their intensities vary drastically along the black cut in Fig. 3(d) while they barely change along the white cut, again suggesting that these peaks are due to the SDW order.

In summary, we have systematically investigated the vortex states in $\mathrm{Ba}_{1-x} \mathrm{~K}_{x} \mathrm{Fe}_{2} \mathrm{As}_{2}$ with the consideration of the interplay between the SDW and ESSC. In the optimally doped compound without induced SDW, there is a NE in-gap peak in the LDOS at the vortex center due to the Adreev bound states, which splits into two asymmetric in-gap peaks when moving away from the center. The effect of the induced SDW is mainly to reduce the intensity of the NE in-gap peak and transfer the spectral weight to form additional peaks outside the gap. For the underdoped sample where the SDW coexists with the ESSC, the vortex cores are dragged towards each other along the $\hat{x}$ direction and the intensity of the NE in-gap peak is further reduced. The obtained result at $x=0.4$ without induced SDW is in qualitative agreement with experiment and we propose future experiments on the near optimally doped and underdoped samples to verify the effect of the SDW on the vortex states. On the other hand, the disappearance of the Adreev bound states in electron-doped $\mathrm{Ba}\left(\mathrm{Fe}_{1-x} \mathrm{Co}_{x}\right)_{2} \mathrm{As}_{2}$ may be due to the induction of strong SDW order in the vortex states, which also needs to be verified by future experiments.

Acknowledgments We thank D. G. Zhang, T. Zhou, C. H. Li, S. H. Pan and A. Li for helpful discussions. This work was supported by the Texas Center for Superconductivity and the Robert A. Welch Foundation under grant numbers E-1070 (Y. Gao and W. P. Su) and E-1146 (H. X. Huang and C. S. Ting).

[1] Y. Kamihara, T. Watanabe, M. Hirano, and H. Hosono, J. Am. Chem. Soc. 130, 3296 (2008).

[2] P. A. Lee, N. Nagaosa, and X. -G. Wen, Rev. Mod. Phys. 78, 17 (2006), and references therein.

[3] M. Rotter, M. Tegel, and D. Johrendt, Phys. Rev. Lett. 101, 107006 (2008).

[4] H. Chen et al., Europhys. Lett. 85, 17006 (2009); R. R. Urbano et al., arXiv:1005.3718 (2010).

[5] G. Wu et al., Europhys. Lett. 84, 27010 (2008); M. Rotter et al., Phys. Rev. B 78, 020503(R) (2008).

[6] C. Liu et al., Phys. Rev. Lett. 101, 177005 (2008); K. Nakayama et al., Europhys. Lett. 85, 67002 (2009); D. V. Evtushinsky et al., Phys. Rev. B 79, 054517 (2009); K. Hashimoto et al., Phys. Rev. Lett. 102, 017002 (2009). 
[7] H. Ding et al., Europhys. Lett. 83, 47001 (2008); X. G. Luo et al., Phys. Rev. B 80, 140503(R) (2009); R. T. Gordon et al., Phys. Rev. Lett. 102, 127004 (2009).

[8] H.-J. Grafe et al., Phys. Rev. Lett. 101, 047003 (2008);

[9] J. D. Fletcher et al., Phys. Rev. Lett. 102, 147001 (2009); C. W. Hicks et al., Phys. Rev. Lett. 103, 127003 (2009).

[10] Y. Nakai et al., Phys. Rev. B 81, 020503(R) (2010).

[11] I. I. Mazin et al., Phys. Rev. Lett. 101, 057003 (2008); Z.-J. Yao, J.-X. Li, and Z. D. Wang, New J. Phys. 11, 025009 (2009); F. Wang et al., Phys. Rev. Lett. 102, 047005 (2009).

[12] L. Shan et al., arXiv:1005.4038 (2010).

[13] Y. Yin et al., Phys. Rev. Lett. 102, 097002 (2009).
[14] X. Hu, C. S. Ting, and J. X. Zhu, Phys. Rev. B 80, 014523 (2009); H. M. Jiang, J. X. Li, and Z. D. Wang, Phys. Rev. B 80, 134505 (2009).

[15] Degang Zhang, Phys. Rev. Lett. 104, 089702 (2010).

[16] T. Zhou, Degang Zhang, and C. S. Ting, Phys. Rev. B 81, 052506 (2010).

[17] A. M. Oleś et al., Phys. Rev. B 72, 214431 (2005).

[18] Y. Sekiba et al., New J. Phys. 11, 025020 (2009).

[19] S. H. Pan et al., private communication.

[20] Hong-Yi Chen and C. S. Ting, Phys. Rev. B 68, 212502 (2003). 